\title{
Entropy and Vorticity Wave Generation in Realistic Gas Turbine Combustors
}

\author{
Bernhard Semlitsch* and Tom Hynes ${ }^{\dagger}$ \\ Whittle Laboratory, Department of Engineering, University of Cambridge, \\ 1 JJ Thomson Avenue, Cambridge, CB3 ODY, United Kingdom \\ Ivan Langella $\ddagger$ and Nedunchezhian Swaminathan ${ }^{\S}$ \\ Hopkinson Laboratory, Department of Engineering, University of Cambridge, \\ Trumpington Street, Cambridge, CB2 1PZ, United Kingdom \\ Ann P. Dowling \\ Department of Engineering, University of Cambridge, \\ Trumpington Street, Cambridge, CB2 1PZ, United Kingdom
}

\begin{abstract}
Understanding the nature of the unsteady flow at the combustor exit is required to accurately simulate time dependent phenomena in the turbine entry, such as indirect noise generation. Using Large Eddy Simulations of the combustion process in a realistic geometry, we analyse the flow at its exit. Two realistic, near-ground certification operating conditions are considered. Different mechanisms for large-scale flow and thermal structure generation are described, which are ejected into the turbine. Modal decomposition methods are used to extract the spatial and temporal scales at the turbine entry. We find that, depending on the operating condition, the entropy waves convect as elongated streaks in the core of the combustor annulus or the proximity of the walls. The dominant unsteady character of the fluctuations exhibits different spectral properties, i.e. low-frequency in the core and high-frequency towards walls. At the combustor exit, the vortical field is dominated by the swirl in the air inlet, which is found to have little influence on the entropy perturbations. Further, the importance of considering the interaction of multiple fuel injectors and combustion zones in an annular combustor is investigated. It is shown that pulsating circumferential vorticity modes can occur in multi-sector annular combustors but these, however, do not affect the entropy wave distribution.
\end{abstract}

\section{Introduction}

Lean combustion is favoured due to its low $\mathrm{NO}_{x}$ emission potential[1]. The advantage of this combustion mode comes with challenges, such as increased unsteadiness, intensified core noise 2 4, and sensitivity to

\footnotetext{
${ }^{*}$ Research Associate, Department of Engineering, University of Cambridge, 1 JJ Thomson Avenue, Cambridge, CB3 0DY, United Kingdom

${ }^{\dagger}$ Reader, Department of Engineering, University of Cambridge, 1 JJ Thomson Avenue, Cambridge, CB3 0DY, United Kingdom

${ }_{\ddagger}$ Lecturer, Department of Aeronautical and Automotive Engineering, Loughborough University, Epinal Way, Loughborough, LE11 3TU, United Kingdom

§Professor, Department of Engineering, University of Cambridge,Trumpington Street, Cambridge, CB2 1PZ, United Kingdom

I Professor, Department of Engineering, University of Cambridge,Trumpington Street, Cambridge, CB2 1PZ, United Kingdom
} 
thermo-acoustic instabilities [1. Hence, its application to practical systems demands development to reduce these drawbacks.

In practical applications, the reactant of lean flames is partially premixed to prevent potential flame flashback. Therefore, fuel/air mixtures with spatiotemporal inhomogeneities arrive at the lean flame front causing unsteady heat release rate and burning rate speed alterations. This leads to amplified pressure, turbulence, and temperature fluctuations, which propagate through the combustion chamber as acoustic, vortical, and entropy waves. Acoustic waves arising from turbulent mixing and volumetric expansion at the flame are referred to as direct noise. Entropy and vorticity waves can cause indirect acoustic noise when accelerated or strained during propagation through turbine stages [5, 6]. Only recently, De Domenico et al. [7] shed some light on the relative importance of these individual noise sources by separating the contributions of direct and indirect noise in an experimental setup. Poinsot 8 highlighted that additional complexities arise in practical combustors. For example, the sophisticated geometry, operating conditions and harsh environment in these combustors differ significantly from laboratory burners. All these factors contribute to the particular transmission properties in gas turbines, where direct noise can be damped while indirect noise is generated during propagation. Therefore, all aspects of core noise need to be considered for acoustic noise predictions of realistic gas turbines.

Different strategies have been employed to estimate the indirect noise generation as disturbances propagate through the turbine ranging from analytical modelling [5] to three-dimensional compressible flow simulations 6 , 9. Analytical approaches focused initially on the one-dimensional transmission properties of compact nozzles [5, 10] and were extended to non-compact nozzles[11] with two-dimensional domains [12] including shock waves [13]. Being computationally efficient, the propagation of vorticity, pressure, and entropy waves through the turbine stages is commonly realised using actuator disk theory allowing the consideration of the entire turbine in the low-frequency range. Realistic wave amplitudes extracted from detailed combustion simulations have been utilised as inlet boundary conditions for low-order models [14]. Mishra and Bodony [15] demonstrated that the actuator disk model is reasonably accurate for cut-on (propagating) waves, while performing inadequately for cut-off (non-propagating disturbance) waves. They showed further that the shape and location of the entropy waves influence the amplitude of acoustic noise generated, which is currently not taken into account by low-order models. Therefore, three-dimensional compressible flow simulations have been used to investigate the impact of non-uniform temperature distributions on the propagation in turbine blade rows. $\mathrm{Mu}$ et al. [9] reported a significant turbine noise amplification with a non-uniform mean temperature pattern mimicking individual burners. Transient entropy waves being entrained into turbines are elongated by the acceleration forming streaks. Holewa et al.16] performed frequency domain simulations (based on the harmonic balance method), where such steady hot temperature streaks imposed at the inlet 
interact with the blade rows. The temperature inhomogeneity was found to decrease the acoustic noise compared to the homogeneous reference case. Gaetani and Persico [17] showed experimentally that steady hot streaks are significantly attenuated over the nozzle guide vanes, while the vorticity field is highly altered.

The contradictory reported results, where entropy waves lead to an overall acoustic noise attenuation[16] or additional noise source [7, 15, 18, are inconclusive having considered only partially the nature of incoming entropy waves and the broadband noise at low frequencies. All investigations employed idealised entropy wave shapes, i.e. spots[15, 17] or plane waves [9]. Further, investigation treated vorticity and entropy waves separately. However, their interaction might be of importance, because the vortical structures might redistribute the hot structures[6]. Thus, realistic entropy wave shapes need to be fed into three-dimensional turbine transmission simulations to clarify the effect of indirect noise. Therefore, we characterise the entropy and vorticity waves that could enter the turbine by analysing Large Eddy Simulation (LES) results of reactive flows inside a gas turbine combustor, which was identified by Morgans and Duran [1] to be one of the main challenges to predict entropy noise generation. Our simulations considered a single and double sector of an annular, industrial combustor operating at near-ground conditions. The processes generating large-scale flow structures are analysed using modal decomposition of flows, temperature, and entropy in the combustor and at its exit.

\section{Numerical Simulations of the Combustor}

\section{A. Case setup}

This work is based on an annular Rolls-Royce developmental lean-burn combustor and the double sector analysed in this study is sketched in Fig. 1 The preheated swirled inlet airflow in each individual sector is split into a central pilot stream and a surrounding, main stream. Kerosene is sprayed through injectors in both air streams. The central swirled flow produces a strong recirculation region in the centre of the combustor where hot gases are entrapped and an annular recirculation region which together provide the stabilisation mechanism for a lifted flame. The flame is designed to be sufficiently far away from the injectors to avoid damage, and these arrangements pose challenges for the numerical modelling described in the next subsection. Effusion cooling arrangements are used for the combustor walls along with six film cooling slots as illustrated in Fig. 1. The gases in the combustor chamber are accelerated by the thermal expansion and the converging geometry downstream before entering the nozzle guide vanes feeding the turbine. In our work, we consider both a single sector and a double sector with two adjacent fuel injectors.

Especially in proximity to the ground, i.e. at approach and take-off conditions, the noise emitted by an aircraft may annoy the population living near the airport and is therefore subject to regulations. Among 


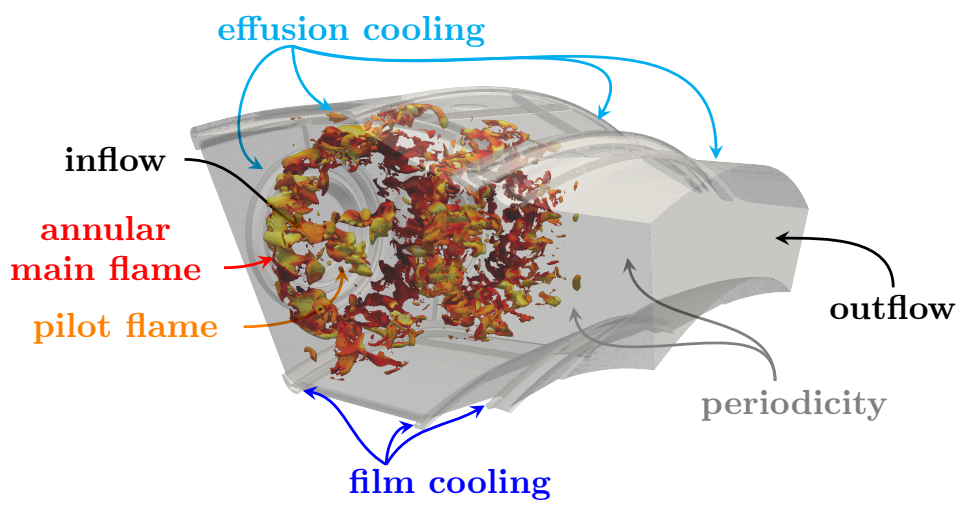

Fig. 1 Sketch of the double-sector combustor geometry with imposed boundary conditions.

various noise sources, engine core noise contributes noticeably [19]. Measures to attenuate this noise are investigated as part of the overall engine performance optimisation. Two scenarios representative of the above two conditions are considered in this study, and these conditions differ from each other in the fuel split between the pilot and main injectors and in the operating pressure. The first condition, namely the Intermediate Pressure Condition (IPC), operates the pilot in rich and the main in lean combustion modes, and the fuel split between pilot and main is $70: 30$. For the second condition, namely the High-Pressure Condition (HPC), the operating pressure is approximately double of the intermediate pressure condition, and both the pilot and main are run in lean combustion mode with a fuel split of $20: 80$, respectively.

\section{B. Numerical Methodology}

\section{Combustion closure}

A flamelet model, known as FlaRe (Flamelet Revised), used in many past works (see for example [20]), is used to model the turbulent combustion. Flamelet models assume that the smallest turbulence eddies do not penetrate (or do not have sufficient energy to affect) the inner structure of the flame, which remains unperturbed. Under this condition turbulence and thermochemistry can be treated separately, yielding substantial gain in terms of computational cost. In partially premixed flames, the fuel-air mixing is tracked using the Bilger mixture fraction $\xi$, whose Favre-filtered transport equation is (in Einstein notation)

$$
\frac{\partial \bar{\rho} \widetilde{\xi}}{\partial t}+\frac{\partial \bar{\rho} \widetilde{u}_{i} \widetilde{\xi}}{\partial x_{i}}=\frac{\partial}{\partial x_{i}}\left(\frac{\widetilde{\mu}}{\operatorname{Sc}} \frac{\partial \widetilde{\xi}}{\partial x_{i}}\right)-\frac{\partial}{\partial x_{i}}\left(\bar{\rho} \widetilde{u_{i} \xi}-\bar{\rho} \widetilde{u}_{i} \widetilde{\xi}\right)+\dot{S}_{e}
$$

In the equation above $t$ is the time, $\bar{\rho}$ is the filtered density, $\widetilde{u}_{i}$ is the Favre-filtered velocity component in the direction $i, \mu$ is the dynamic viscosity and $\mathrm{Sc}=0.7$ is the Schmidt number, which is taken to be the same for all scalars (unity Lewis number). The last two terms in Eq. 1 are respectively turbulent transport and 
a source term due to the evaporation of the fuel spray. The turbulent transport term is modelled using a gradient hypothesis, i.e. $\bar{\rho} \widetilde{u_{i} \xi}-\bar{\rho} \widetilde{u_{i}} \widetilde{\xi}=-\left(\mu_{t} / \mathrm{Sc}_{t}\right) \nabla \widetilde{\xi}$, where $\mu_{t}$ is the sub-grid scale (SGS) eddy viscosity and $\mathrm{Sc}_{T}=0.7$ the turbulent Schmidt number, which is taken to be the same for all scalars. The variable used to track the reaction progress is defined as $c=Y_{\mathrm{CO}_{2}} / W_{\mathrm{CO}_{2}}+Y_{\mathrm{CO}} / W_{\mathrm{CO}}$, where $Y_{k}$ and $W_{k}$ are mass fraction and molecular mass of species $k$, and its Favre-filtered transport equation is

$$
\frac{\partial \bar{\rho} \widetilde{c}}{\partial t}+\frac{\partial \bar{\rho} \widetilde{u}_{i} \widetilde{c}}{\partial x_{i}}=\frac{\partial}{\partial x_{i}}\left(\frac{\widetilde{\mu}}{\operatorname{Sc}} \frac{\partial \widetilde{c}}{\partial x_{i}}\right)-\frac{\partial}{\partial x_{i}}\left(\bar{\rho} \widetilde{u_{i} c}-\bar{\rho} \widetilde{u}_{i} \widetilde{c}\right)+\overline{\dot{\omega}}
$$

where the SGS transport term is also modelled with a gradient hypothesis. The filtered reaction rate, $\overline{\dot{\omega}}$, is modelled as:

$$
\overline{\dot{\omega}}=\bar{\rho} \int_{0}^{1} \int_{0}^{1} \frac{\dot{\omega}(\zeta, \eta)}{\rho} P\left(\zeta, \eta ; \widetilde{c}, \widetilde{\xi}, \sigma_{c}^{2}, \sigma_{\xi}^{2}\right) \mathrm{d} \zeta \mathrm{d} \eta
$$

where $\dot{\omega}$ is the flamelet reaction rate from a one dimensional unstrained planar laminar premixed flame of a given mixture fraction $\eta$. The symbols $\zeta$ and $\eta$ are the sample space variables for $c$ and $\xi$. The presumed joint probability density function, $P$, is modelled using the product of two Beta-functions as described by Ruan et al. [21] and Chen et al. [22]. The Beta-functions need the progress variable and mixture fraction SGS variances, $\sigma_{c}^{2}$ and $\sigma_{\xi}^{2}$ and these values are obtained using their respective modelled transport equations. The equation for $\sigma_{\xi}^{2}$ is

$$
\bar{\rho} \frac{D \sigma_{\xi}^{2}}{D t}=\frac{\partial}{\partial x_{i}}\left[\left(\overline{\rho \mathcal{D}}+\frac{\mu_{t}}{S c_{t}}\right) \frac{\partial \sigma_{\xi}^{2}}{\partial x_{i}}\right]-2 \bar{\rho} \widetilde{\varepsilon}_{\xi}+2 \bar{\rho} \frac{\nu_{t}}{S c_{t}}\left(\frac{\partial \tilde{\xi}}{\partial x_{i}} \frac{\partial \tilde{\xi}}{\partial x_{i}}\right)+\dot{S}_{e, \sigma_{\zeta}} .
$$

The SGS scalar dissipation rate, the third term in Eq. 4, is modelled using a linear relaxation model [23], $\widetilde{\varepsilon}_{\xi}=2 \nu_{T} /\left(\mathrm{Sc}_{T} \Delta^{2}\right) \sigma_{\xi}^{2}$. The equation for $\sigma_{c}^{2}$ is

$$
\bar{\rho} \frac{D \sigma_{c}^{2}}{D t}=\frac{\partial}{\partial x_{i}}\left[\left(\overline{\rho \mathcal{D}}+\frac{\mu_{t}}{S c_{t}}\right) \frac{\partial \sigma_{c}^{2}}{\partial x_{i}}\right]-2 \bar{\rho} \widetilde{\varepsilon}_{c}+2 \bar{\rho} \frac{\nu_{t}}{S c_{t}}\left(\frac{\partial \widetilde{c}}{\partial x_{i}} \frac{\partial \widetilde{c}}{\partial x_{i}}\right)+2(\overline{c \dot{\omega}}-\widetilde{c} \overline{\dot{\omega}})
$$

where the reaction term is modelled in a manner similar to Eq. 3. The SGS scalar dissipation rate in this equation cannot be modelled using a simple relaxation model as discussed by Langella \& Swaminathan [24]. Thus, the model of Dunstan et al. [25] with dynamic computation of the combustion parameter [26] is used for this study, and additional details on this closure can be found in these references.

\section{Spray modelling}

The kerosene sprayed at the injectors is modelled using a two-way coupled Eulerian-Lagrangian approach with secondary Schmehl breakup [27, and a Rosin-Rammler distribution for the droplet diameter. The 
droplets are injected at a constant velocity and with a random injection angle sampled using a Gaussian distribution with $10^{\circ}$ standard deviation and mean direction aligned with the injector axis. A rapid mixing formulation is used to model the droplet evaporation, where the liquid kerosene is assumed to have very large thermal conductivity, and its evaporation temperature is set to $650 \mathrm{~K}$.

\section{Computational details}

Reacting, incompressible (density-varying) Navier-Stokes equations are solved using PRECISE-UNS [28, which employs the finite volume approach to discretise the transport equations for mass, momentum and energy, along with the four equations specific for the combustion model, presented in section II.B.1. Using Einstein's index convention, the equations for mass and momentum are:

$$
\begin{gathered}
\frac{\partial \bar{\rho}}{\partial t}+\frac{\partial \bar{\rho} \widetilde{u}_{i}}{\partial x_{i}}=0 ; \quad \text { and } \\
\frac{\partial \bar{\rho} \widetilde{u}_{i}}{\partial t}+\frac{\partial \bar{\rho} \widetilde{u}_{i} \widetilde{u}_{j}}{\partial x_{j}}=-\frac{\partial \bar{p}}{x_{i}}+\frac{\partial}{\partial x_{j}}\left(\widetilde{\mu} \frac{\partial \widetilde{u}_{i}}{\partial x_{j}}\right)-\frac{\partial \tau_{i j}^{r}}{\partial x_{j}}
\end{gathered}
$$

where $\bar{p}$ is the modified filtered pressure, which is the sum of filtered pressure and $2 k / 3$, with $\mathrm{k}$ as the SGS kinetic energy [29]. The SIMPLEC algorithm [30] is used for the pressure-velocity coupling. The last term in the momentum equation represents the anisotropic part of the SGS stress tensor, and is modelled using constant Smagorinsky model as $\tau_{i j}^{r}=-2 \mu_{t} \widetilde{S}_{i j}$, where the eddy viscosity is $\mu_{t}=\bar{\rho}\left(C_{s} \Delta\right)^{2}|\widetilde{S}|$ with $C_{s}=0.17 .|\widetilde{S}|$ is the magnitude of the resolved strain tensor. The filtered kinematic viscosity is obtained using Sutherland's law, $\widetilde{\mu}=A_{s} \sqrt{\widetilde{T}} /\left(1+T_{s} / \widetilde{T}\right)$, with $A_{s}=1.67 \times 10^{-6}$ being a dimensional constant and $T s=170.7 \mathrm{~K}$.

A transport equation for the Favre-filtered enthalpy $\widetilde{h}$ (sum of sensible and formation enthalpies) is used as energy equation to account for sub-adiabaticity and mixing between different streams, and this equation is

$$
\frac{\partial \bar{\rho} \widetilde{h}}{\partial t}+\frac{\partial \bar{\rho} \widetilde{u}_{i} \widetilde{h}}{\partial x_{i}}=\frac{\partial}{\partial x_{i}}\left(\frac{\widetilde{\mu}}{\operatorname{Sc}} \frac{\partial \widetilde{x i}}{\partial x_{i}}\right)-\frac{\partial}{\partial x_{i}}\left(\widetilde{\rho} \widetilde{u_{i} h}-\bar{\rho} \widetilde{u}_{i} \widetilde{h}\right)
$$

where the latter term is modelled using a gradient hypothesis as for the other scalars described in section II.B.1. and the pressure variation term has been excluded because of the low-Mach hypothesis. The Favre-filtered temperature is taken from the flamelet table and corrected using the enthalpy from the simulation as $\widetilde{T}=\widetilde{T}_{f}+\left(\widetilde{h}-\widetilde{h}_{f}\right) / \widetilde{C}_{p}$, where $\widetilde{T}_{f}$ is the temperature from the flamelet and $\widetilde{h}_{f}$ and $\widetilde{C}_{p}$ are respectively flamelet enthalpy and the specific heat capacity at constant pressure at temperature $T_{f}$. These are computed a priori in a manner similar to Eq. (3), and are stored in a lookup table. The density is computed from the operating 
pressure and local temperature using the state equation for perfect gases and it is assumed to be constant for the liquid kerosene.

The transport equations are discretised using second-order central schemes for the spacial gradients. A second-order scheme is also used for time derivatives, and a relaxation factor of 0.7 is used for all transported variables except for pressure whose relaxation factor is 0.4 . The time step for all simulations is $\Delta t=0.5 \mu \mathrm{s}$. This guarantees maximal CFL numbers of 0.4 overall in the combustor except for regions close to walls and injectors, where the CFL criterion is allowed to be higher to make the computational cost affordable. A maximum of 10 sub-steps is used for each time step to reach convergence in the SIMPLEC loop. Also, the velocity gradients across the flame are limited using a Van Albada limiter.

The flamelet quantities are computed using the Dagaut mechanism [31] and Chem1d, which solves the one-dimensional freely propagating flames on adaptive grid using finite difference technique for a number of mixture fraction values within the flammability limits. The flamelet quantities are then integrated as described earlier and stored in a 4-D look-up table. Equispaced 100, 200 and 50 points over the ranges of the controlling variables $\widetilde{c}, \widetilde{\xi}$ and $\sigma_{c}^{2}$ are used while generating the look-up table. For $\sigma_{\xi}^{2}, 15$ points are spaced exponentially (with an exponential factor of 2.5) in the look-up table to cluster more points close to zero, because the SGS mixture fraction variance in the flame region is caused only by evaporation (resulting in small values for the case under study).

The numerical mesh is hexa-dominant with a hexa core and tetrahedra in the wall region. This choice was observed in preliminary tests to reduce the mesh size for a fixed accuracy using PRECISE-UNS, and results in about 13 million and 26 million elements respectively for the single and double sector cases. An additional mesh of 9 million elements, differing mainly in the region of the flame, was used for preliminary calculations and mesh sensitivity for the single sector case. In particular, a detailed analysis was conducted to assess the mesh quality using the Pope's criterion [29], which states that the ratio $\mathcal{Q}=k /(k+\mathcal{K})$ between SGS and total (SGS plus resolved) turbulent kinetic energy has to be smaller than $20 \%$ in a well resolved LES. The analysis was conducted on both preliminary and final mesh sizes, and for both the intermediate and high power conditions discussed in this work. The SGS kinetic energy, $k$, was estimated locally using the Lilly's model [32], $k=\mu_{t}^{2} /\left(C_{L} \bar{\rho} \Delta\right)^{2}$, with $C_{L}=0.1$. Typical contours of mean $\mathcal{Q}$ on the fine mesh are shown for the intermediate pressure condition in Fig. 2. It is shown that Pope's criterion is satisfied everywhere in the domain except for the region near the walls. This is expected as the boundary layer is not resolved. This does not affect the specific results in this work, because the substantial proportion of the turbulence is generated by shear layers in the combustor. As the Smagorinsky model is used for $\mu_{t}, k$ does not go to zero in the laminar regions upstream and near the wall. It is worth noting that the $20 \%$ criterion introduced by Pope [29] is for non-reacting flows, and thus its evaluation in regions affected by heat release (which would 
locally increase the instantaneous value of $k$ ) has to be mindful. In addition to the analysis above, first and second order statistics relevant for the analysis to be conducted in section III and obtained using the 9 million and 13 million cell meshes (both related to the single sector geometry), were also compared to each other and no significant difference could be observed, suggesting that this grid size is satisfactory for the analysis conducted in this work.

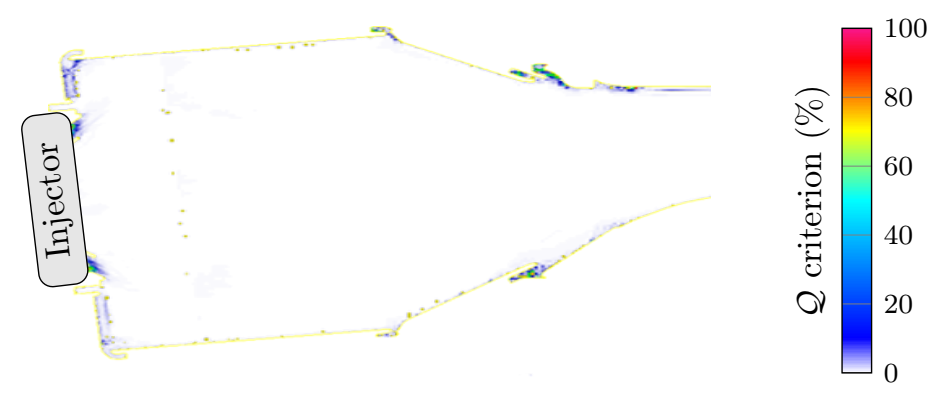

Fig. 2 Pope's criterion is shown in mid-plane view for the intermediate pressure condition.

Boundary conditions are assigned as follows: mass flow rates are assigned at the inlets (air stream, film and effusion cooling), while all other scalars are assigned to have zero normal gradients. Enthalpies at the inlets are assigned to be consistent with the temperature and rate of mixing of the incoming stream. Progress variable, mixture fraction and their SGS variances are assigned to be zero at all inlets. At the outlet, all transported quantities are assigned a zero-gradient condition except for pressure which has a fixed value. At the adiabatic walls, the velocity is zero, and all scalars have a zero-gradient condition. No special treatment is used at the walls for the velocity field due to the high velocities of the studied configuration (order of 250 $\mathrm{m} / \mathrm{s}$ in the reactants), as this would require a significant increase of mesh size. This is acceptable near the injector walls as discussed above, where most of the turbulence is produced by the shear layers and the swirl. Close to the combustor walls temperature hot spots are present in the high-pressure condition as will be shown later, and thus their position could be slightly affected by the assumptions at the wall. However, these high-temperature spots occur far enough from the wall so that several numerical cells are still present in between, and thus the above assumptions do not affect the analysis in the present work.

A single sector simulation for about $60 \mathrm{~ms}$ of physical time (for the $13 \mathrm{M}$ grid) took about 21 days, using 512 cores on the Darwin cluster in Cambridge. About $20 \mathrm{~ms}$ are needed for the initial transient in the LES, and additional $40 \mathrm{~ms}$ simulation time are used for data sampling to resolve fluctuations down to a frequency of $25 \mathrm{~Hz}$ for the spectral analysis discussed in the next section. The double sector simulations took about 23 days using the same number of cores for about $50 \mathrm{~ms}$ (40 ms sampling and $10 \mathrm{~ms}$ transient), but using the newer and more efficient CSD3 cluster in Cambridge. 


\section{Modal decomposition analysis and post-processing}

Interpreting instantaneous manifestations of the flow may not be statistically significant. Hence, coherent large-scale flow structures are extracted through proper orthogonal mode decomposition using 793 (IPC) and 804 (HPC) running-averaged realisations of the entropy, $\widetilde{s}$, and velocity field, $\widetilde{u}$, sampled at $20 \mathrm{kHz}$ in the entire computational domain. * The use of running-averaged data (averaged in between sample intervals) instead of flow-field instances filters high-frequencies, which would be spuriously aliased into the frequency range of interest.

A short description of the proper orthogonal decomposition method is provided, while a detailed introduction has been provided by Chatterjee [33]. The sampled data, $\widetilde{s}=\widetilde{s}\left(t_{1}\right), \widetilde{s}\left(t_{2}\right), \ldots, \widetilde{s}\left(t_{j}\right)$, is decomposed into a series of spatial, $\phi_{j}(x)$, and temporal functions, $a_{j}(t)$ to optimally represent, in least square sense, the system behaviour. This is achieved by using the auto-covariance of the decomposed quantity, i.e. entropy or velocity, to formulate the least square optimisation problem

$$
\min _{\phi_{j}}\left(\widetilde{s}(x, t)-(\underbrace{a_{0}(t) \phi_{0}(x)}_{\text {mean }}+\underbrace{\sum_{j=1}^{J} a_{j}(t) \phi_{j}(x)}_{\text {perturbations }})\right)^{2}
$$

which can be recasted as eigenvalue problem[34],

$$
R \varphi_{j}=\lambda_{j} \varphi_{j} \quad \text { where } \quad R=\sum_{j=1}^{J} \widetilde{s}_{j} \widetilde{s}_{j}
$$

where $\varphi_{j}$ is the spatial eigenfunction and $\lambda_{j}$ is the associated eigenvalue. The eigenvector are the temporal modes, $a_{j}$ and the spatial modes, $\phi_{j}$, are reconstructed from the eigenvalues. Hence, the spatial modes are orthogonal. This allows separate analyses of the characteristic scales, since the stochastic evolution is decomposed into temporal $a_{j}(t)$ and spatial $\phi_{j}(x)$ components. The zeroth-order mode represents the mean and the higher-order modes describe the perturbations on top of the mean.

\section{Results - Flow characterisation}

The fuel split governs the heat release rate and temperature distribution in the gas turbine combustor, which can change significantly with the operating condition. At the intermediate pressure condition, the predominant heat release occurs at the central pilot flame as illustrated in Fig. 3 a), whereas the heat release

${ }^{*}$ The entropy is calculated by the relation, $\widetilde{s}=\widetilde{c_{p}} / \gamma \cdot \ln \left(\frac{\gamma-1}{\gamma} \widetilde{c_{p}} \rho^{1-\gamma} \widetilde{T}\right)$, where $\widetilde{c_{p}}$ is the specific heat capacity, $\gamma$ is the heat capacity ratio and $\widetilde{T}$ is the local temperature. 

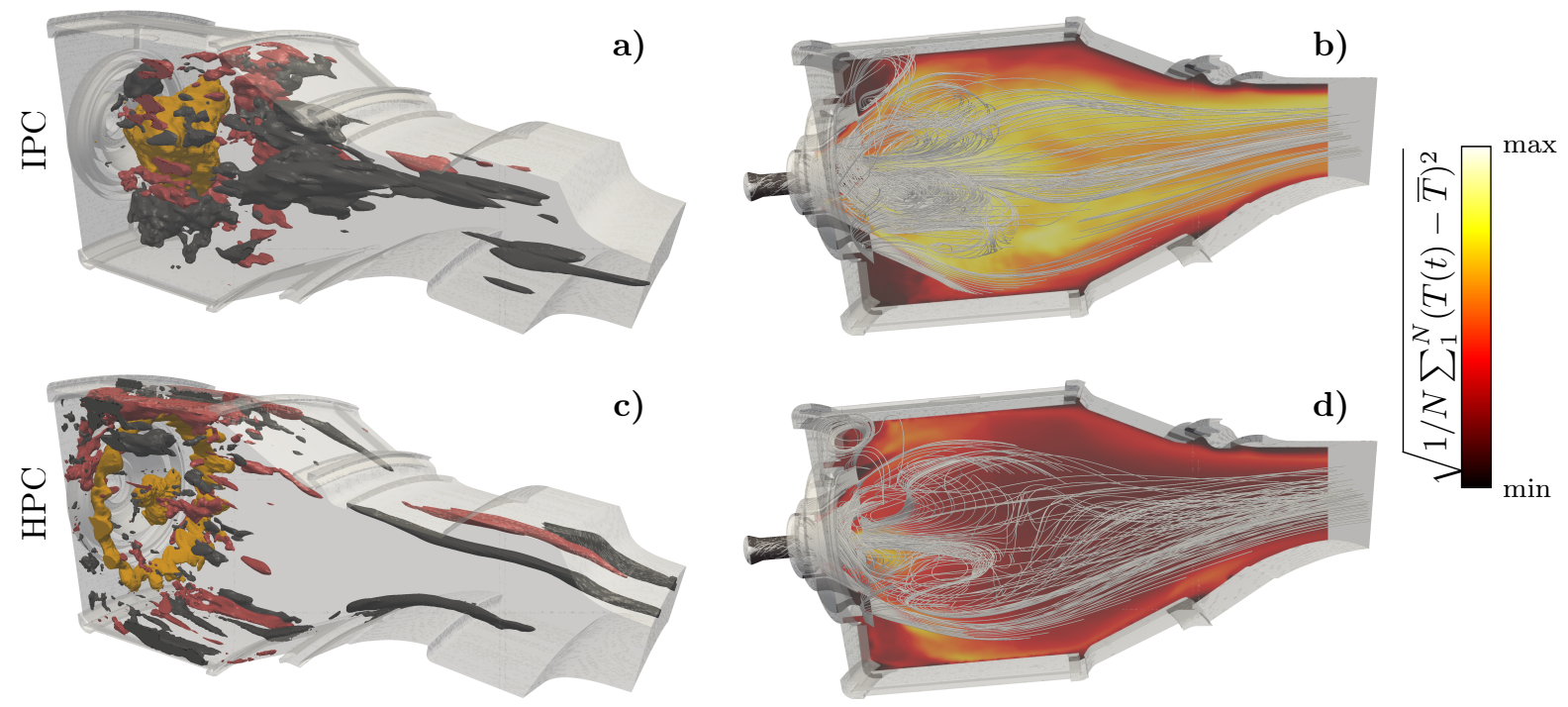

Fig. 3 To the left, the locations of entropy wave generation in terms of temperature perturbation modes. Black and red coloured structures correspond to high and low temperature spots. The time-averaged flame is illustrated in yellow. To the right, the streamlines illustrate the time-averaged flow pattern, where the root-mean-square of the temperatures is plotted in the mid-plane.

is redistributed towards the annular main flame for the high-pressure operating condition shown in Fig. 3 b). Entropy waves are generated primarily by the lean flame, hot combustion gas mixing with the cooling flow, and the interaction of the main with pilot flame. Figure 3 b) shows that the entropy waves with the highest amplitudes are produced in the proximity of the effusion and film cooled combustion chamber walls for the high-pressure operating condition. These entropy waves convect as streaks along the vicinity of the walls towards the turbine. Less fuel arrives at the main flame at the intermediate pressure condition and therefore, the flame remains shorter. Therefore, it merges with the pilot flame and does not reach the combustor walls. With the interaction of main and pilot flame, the largest coherent entropy structures are generated for this operating condition. The streamlines in Fig. 3 b) and d) reveal large recirculation zones in the centre of the combustor (for both operating conditions). Consequently, entropy propagation along the centreline is avoided and hence the temperature fluctuations are low downstream of the pilot flame (as shown in Fig. 3 b) and d) ). The large coherent entropy structures are recirculated at the intermediate pressure operating condition and convect thereafter to the mid-height of the converging duct.

\section{A. Averaged Flow Characteristics at the Combustor Exit}

These generation mechanisms of the coherent entropy wave propagation cause distinct fluctuation patterns at the combustor exit as shown in Fig. 4. In time-average, a high-temperature circumferential band manifests 


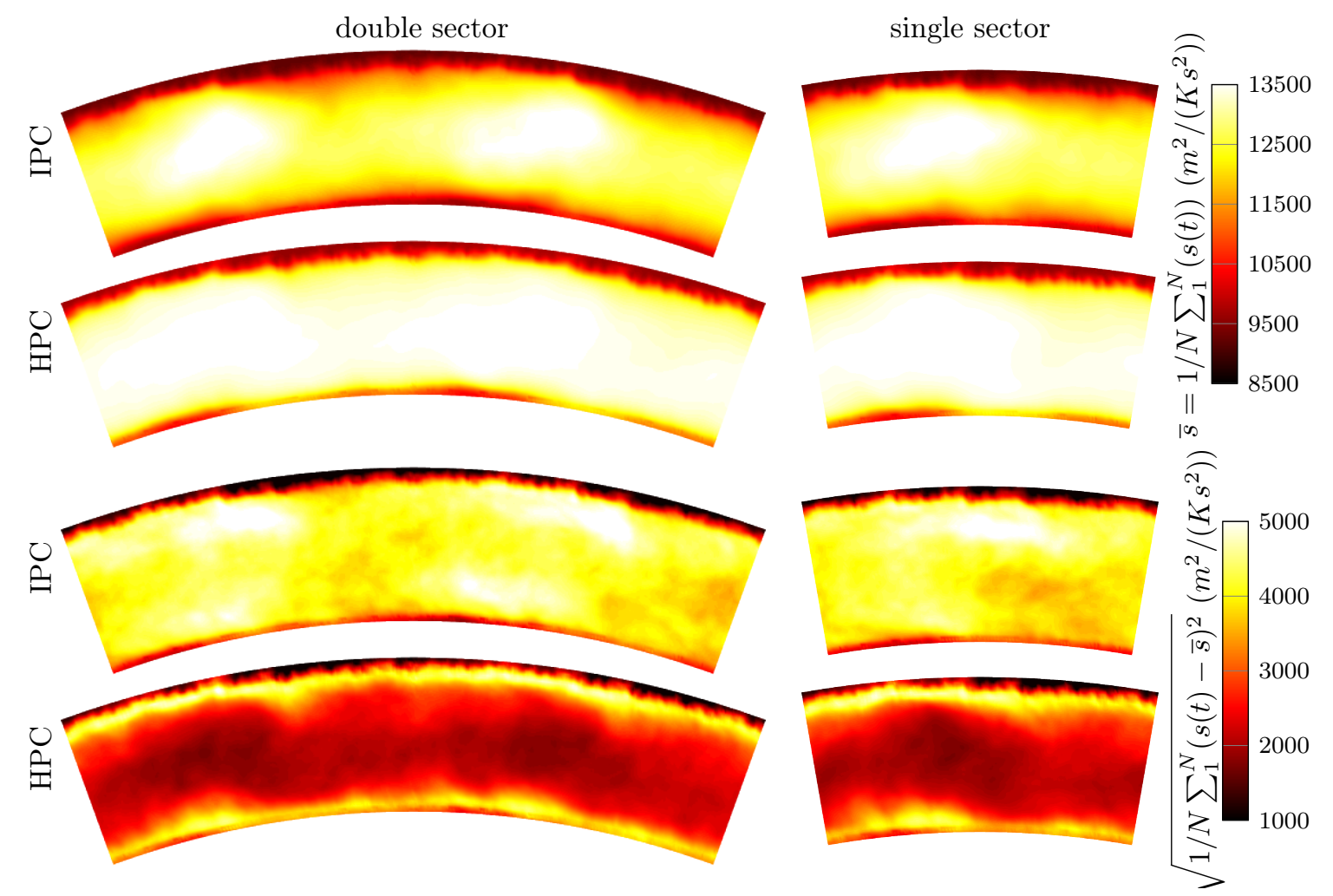

Fig. 4 Characterisation of the time-averaged flow in terms of entropy, $s=c_{p} / \gamma \ln \left(p / \rho^{\gamma}\right)$, and its root-mean-square statistics at the combustor exit for the two operating conditions.

at the high-pressure operating condition, whereas a hot, slightly off-centred spot can be observed at the intermediate pressure operating conditions. The combustor surfaces are protected by effusion and film cooling and hence, low temperatures are observed towards the walls for both operating conditions. This steep radial temperature gradient in the proximity of the walls causes high-temperature variations with the mixing and alternating convection of hot combustion and cold cooling gasses. Figure 4 reveals that substantial temperature fluctuations establish in the mid-height at the intermediate pressure condition, while significantly lower fluctuations are present in the core flow for the high-pressure operating condition.

Although the temperature field at the combustor exit varies within the investigated operating conditions, Fig. 5 reveals that the time-averaged flow field is qualitatively similar. Nonetheless, the Mach-number fluctuations are reduced at the intermediate pressure condition. The in-plane Mach number components of the time-averaged flow field reveal a large-scale circulation originating from the swirled inlet flow. In contrast to the peak temperatures, the vortex core is slightly shifted to the right side. The importance of this swirl is supported by the shape of the velocity fluctuations (characterised by the in-plane root-mean-square in Fig. 57. This centred vortical structure disappears at times during the intermediate pressure condition, while being present continuously for the high-pressure operating condition. This explains the reduced in-plane 

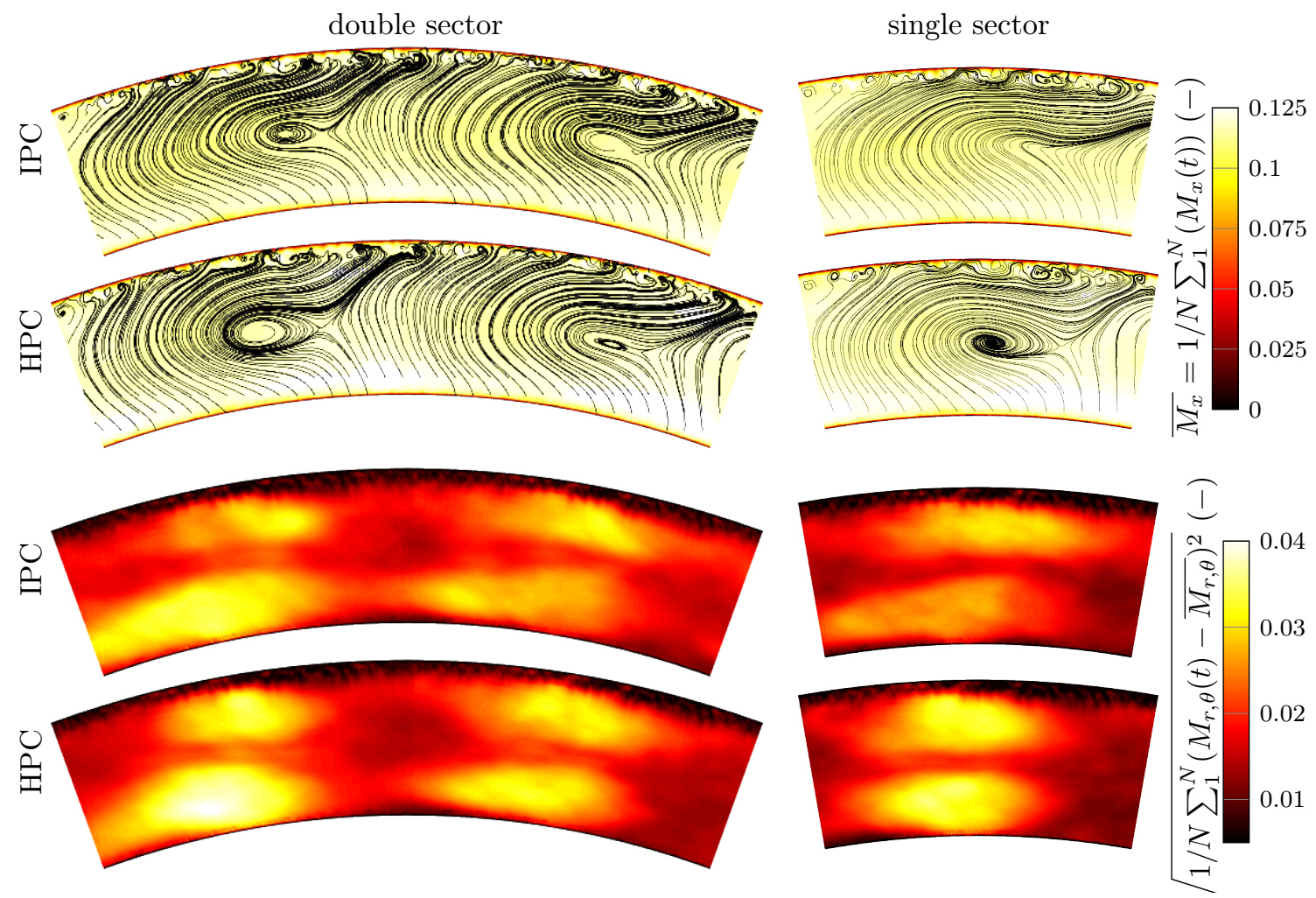

Fig. 5 Characterisation of the time-averaged flow in terms of Mach number components, $M_{x, r, \theta}$, and its root-mean-square statistics at the combustor exit for the two operating conditions.

velocity fluctuations observed at the intermediate pressure condition. A radial gradient of the axial velocity component at the combustor exit is caused by the steeper slope of the geometrical convergence at the inner wall.

\section{B. Wave Perturbations at the Combustor Exit}

To analyse the temporal behaviour of the perturbations in the combustor exit plane, the spectra of the radially and circumferentially integrated quantities are calculated and shown in Fig. 6. These reveal a broadband character for the fluctuations of the streamwise vorticity and entropy waves at the combustor exit, where only insignificant deviations between single and double sector simulations can be observed. In accordance with the spatial fluctuation amplitudes illustrated in Fig. 5 , the amplitudes of the entropy spectra differ between operating conditions, while only minor variations can be observed for the vorticity spectra.

The impact of the combustion vortex on the entropy redistribution is analysed via the correlation coefficient, $R_{u, s, \text { coeff }}$, which is defined as the cross-correlation between entropy and vorticity waves normalised by the autocorrelations at zero time lag; $R_{u, s, c o e f f}(t)=R_{u, s}(t) / \sqrt{R_{u, u}(0) R_{s, s}(0)}$. For the computation, the quantities on the exit planes of the combustor sectors are integrated (radially and circumferentially) and 


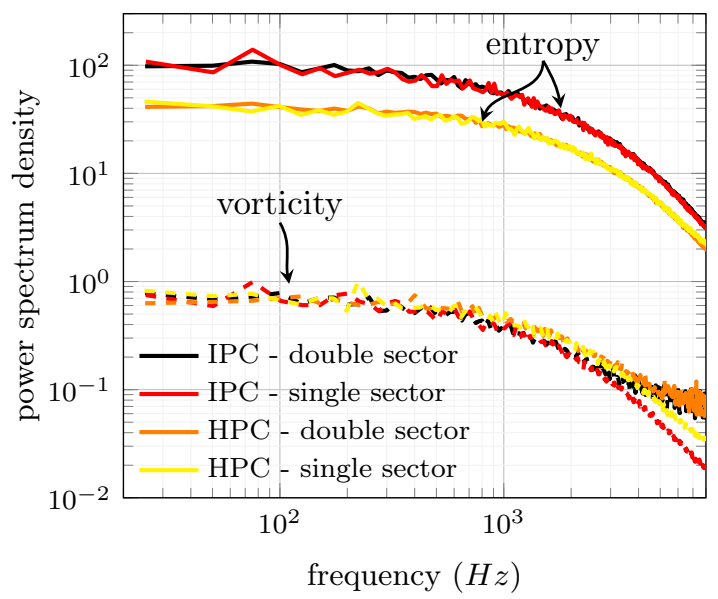

Fig. 6 Spectra of the vorticity and entropy fluctuations at the combustor exit plane are plotted.

a) intermediate pressure condition

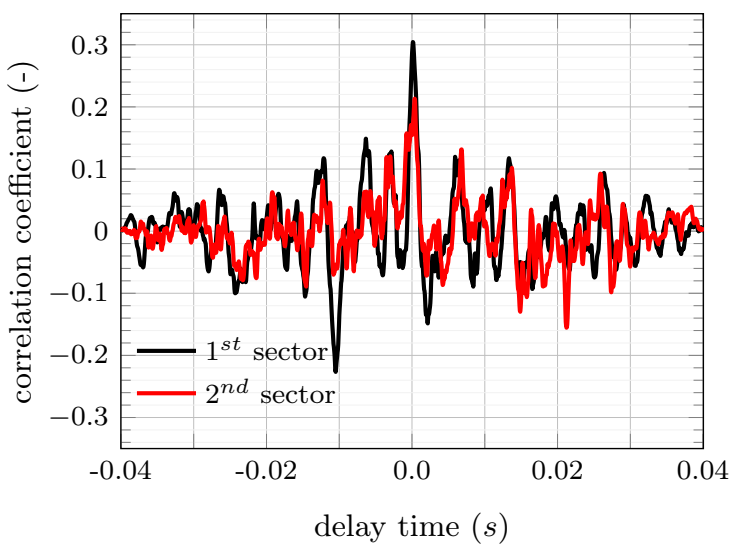

b) high-pressure condition

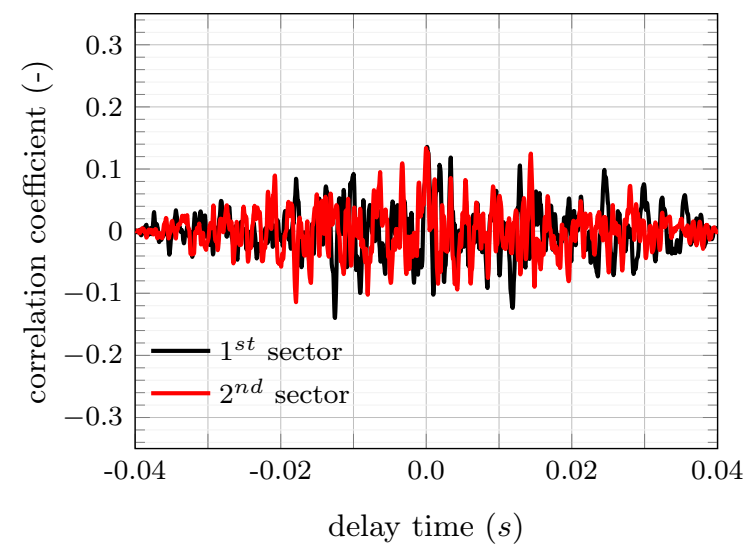

Fig. 7 The cross-correlation between the vorticity and entropy are shown for the individual sectors of the double-sector simulation.

evaluated individually. Figure 7 a) reveals the correlation between vorticity and entropy perturbations for the intermediate pressure operating condition. The correlation is expected because the entropy perturbations convect for this operating condition near the mid-passage where the combustion vortex affects the flow. For the high-pressure operating condition, the entropy perturbations convect close to the cooling flow near the walls, where the large flow structures have less impact. Therefore, the correlation between vorticity and entropy perturbations is shown to be less significant for the high-pressure operating condition in Fig. 7 b).

The correlation coefficients, $R_{u 1, u 2, \text { coeff }}$ and $R_{s 1, s 2, \text { coeff }}$, based on the integrated quantities between the individual combustor sector exit areas provides an estimate for the interaction between fuel injectors. A dominant out-of-phase relation between the streamwise velocity fluctuations in the individual sectors of the double-sector simulation can be observed even of crude averaging over the combustor exit plane, as shown in 

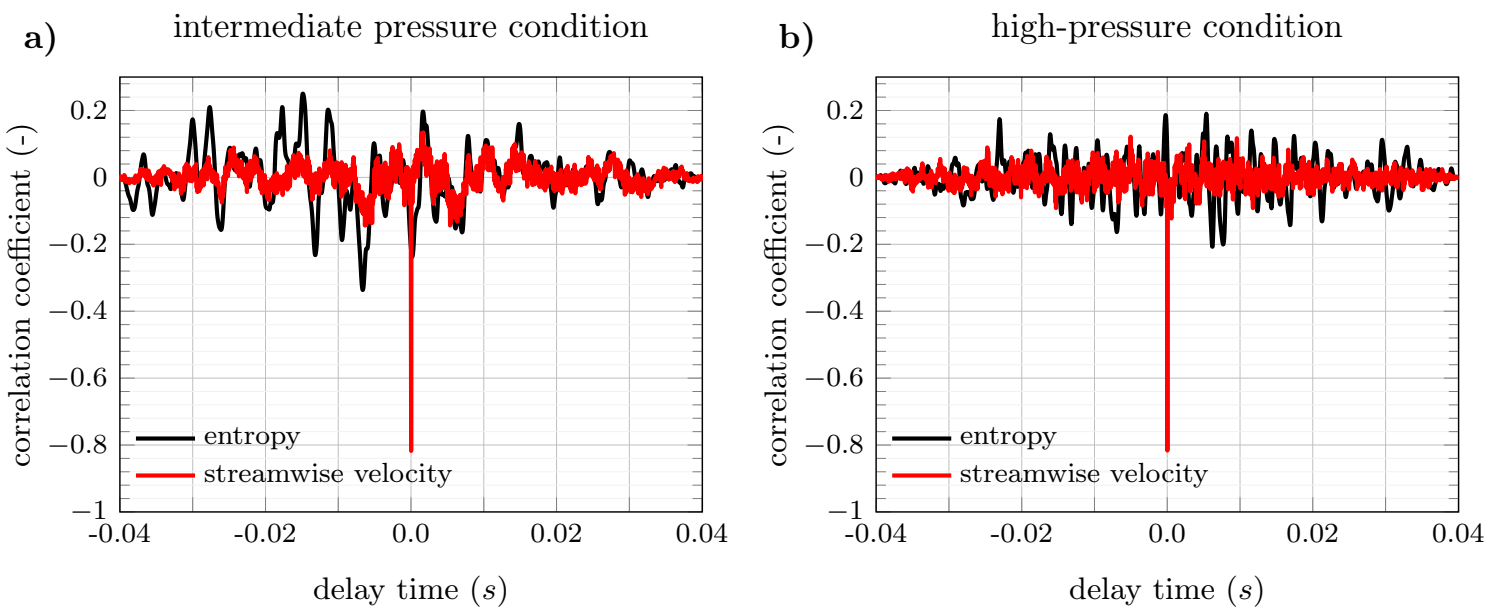

Fig. 8 The cross-correlations of the quantities in between sectors of the double-sector simulation are shown.

Fig. 8. This phase relationship can be identified in the shapes represented by the $1^{\text {st }}$ and $3^{\text {rd }}$ vortical proper orthogonal modes for the double sector shown in Fig. 9. For the leading proper orthogonal mode, the axial velocity shows opposite signs in the individual sectors. Modulating this spatial shape by the oscillations of the temporal mode, this represents a pulsation in anti phase for the two sectors. The amplitudes of the first and third mode represent a $0.35 \%$ and $0.19 \%$ energy perturbation of the mean flow, respectively. Only the second and higher modes show similar shapes at the combustor exit as observed simulating a single sector, which reveal a horizontally meandering motion of a vortex. These largest coherent cells stretch out over the entire annular gap height.

Figure 10 illustrates that entropy waves exhibit different spatial patterns at the two operating conditions modelled. At the intermediate pressure condition, the coherent entropy streaks expand to the combustor walls, while being even wider in the circumferential direction. At the high-pressure condition, entropy waves with high amplitudes can be primarily observed in the shear-layer between the hot combustion gasses and the cooling flows. Their circumferential length is observed to stretch up to the half of a combustor sector. However, minimal entropy wave amplitudes are notable at mid-radius, and the shapes of the entropy structures are similar for the single and double sector simulations at both operating conditions.

\section{Modelling Aspects}

To analyse the validity of representing entropy waves as planar waves, the sampled sequence is integrated over the radius, $r$, and expanded into circumferential modes,

$$
s_{m, j}=\sum_{m=-\infty}^{\infty} \frac{1}{r_{O}^{2}-r_{I}^{2}} \int_{r_{I}}^{r_{O}} \widetilde{s}(r, \theta, j \Delta t) r d r e^{i m \theta \alpha}
$$



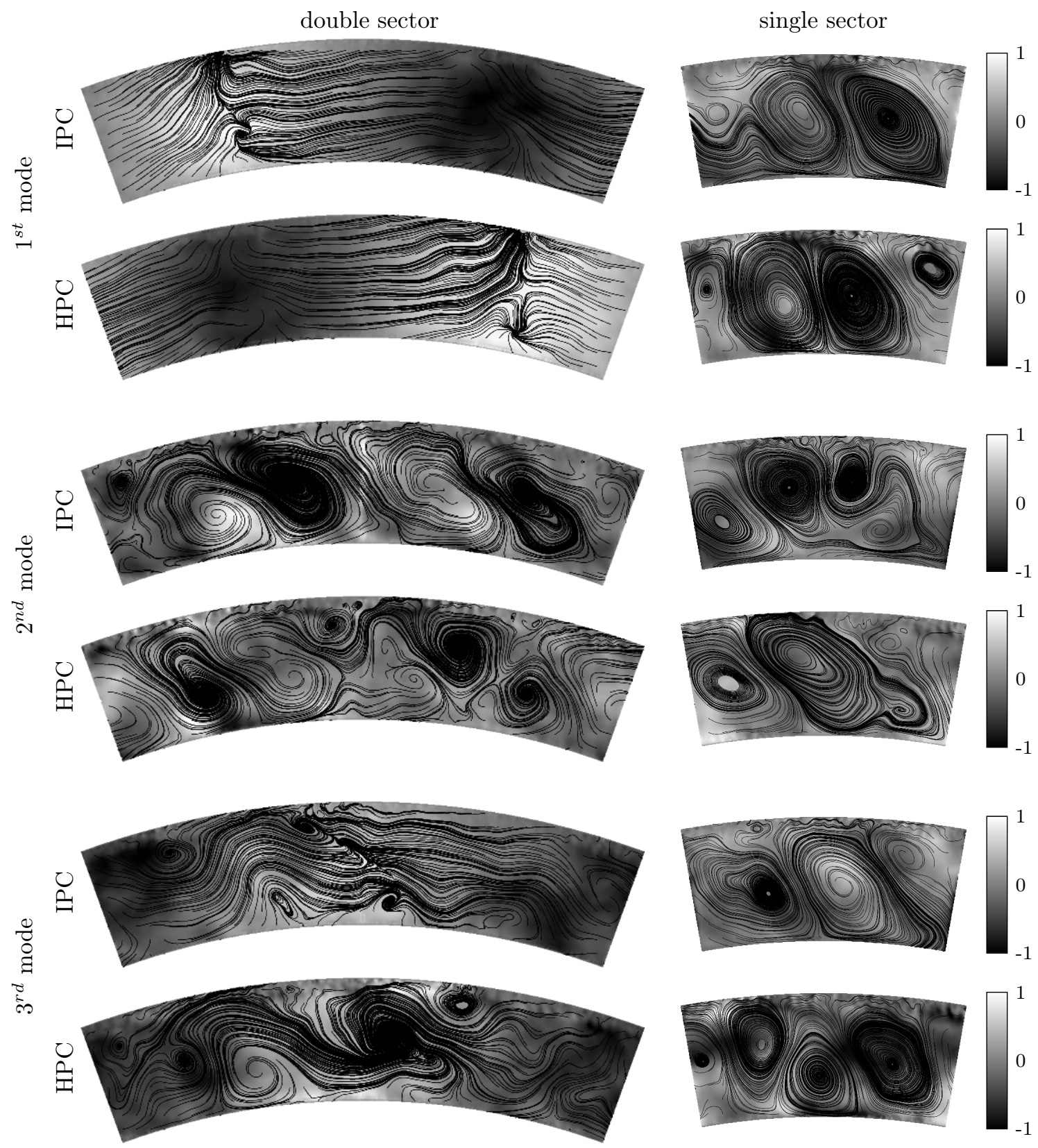

Fig. 9 The normalised vorticity modes are compared for the investigated operating conditions at the combustor exit. The streamlines illustrate the in-plane velocity components and the colourscale represents the axial velocity component normalised by the maximal absolute value.

where $j$ is the sequence index of the sample, $\theta$ is the circumferential coordinate, $m$ is the circumferential mode number, and $\alpha$ is the number of blade passages around the annulus. The indices, $I$ and $O$, denote the inner and outer radius of the annulus. The spectral description is calculated by the discrete Fourier 

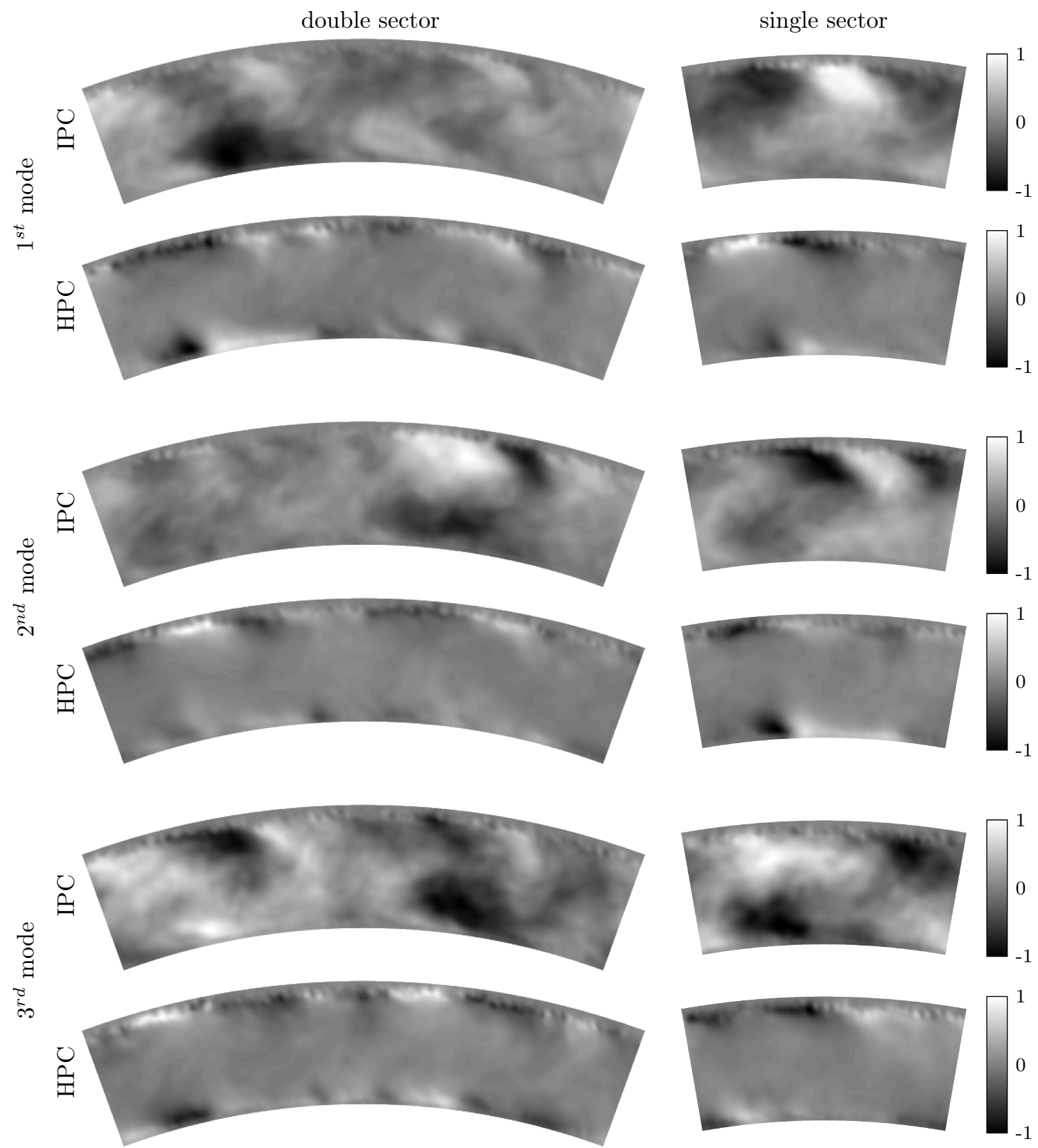

Fig. 10 The entropy perturbation character is shown by the leading proper orthogonal modes at the combustor exit, which are normalise by the maximal absolute value.

transforms of the entropy signal,

$$
\hat{s}_{m}\left(f_{k}\right)=\sum_{n=0}^{J-1} s_{m, j} e^{-2 \pi i k j / J} \quad \text { where } \quad f_{k}=k \Delta f
$$

where $J$ the total sample number, $\Delta f=1 / \Delta t$ is the frequency resolution. Figure 11 shows that planar waves, i.e. $m=0$, are not dominating the entropy fluctuation content, which is rather spread with equal intensity 
over several circumferential mode numbers.
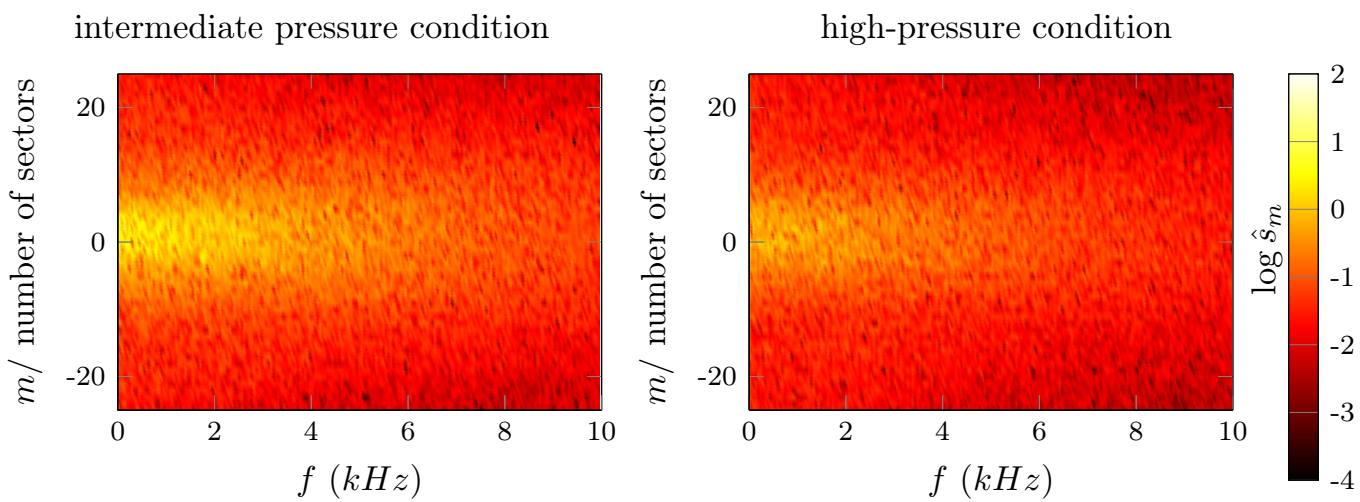

Fig. 11 The entropy wave amplitudes at the combustor exit are shown as funcition of frequency, $f$ and circumferential mode number, $m$.

The proper orthogonal mode decomposition showed additionally that the shape of the unsteadiness in the heat release rate cannot be realistically described by pulsating the time-averaged flow at the combustor exit. As demonstrated, the location of the highest entropy fluctuations does not coincide with the highest mean temperatures. Hence, realistic entropy and vorticity wave interface conditions demand a higher-order model for indirect noise estimations, e.g. a representation via the leading proper orthogonal modes. To estimate the number of proper orthogonal modes required to represent a fraction of the fluctuation content contained in the proper orthogonal analysis, the eigenvalues ratio of the truncated series to the sum of all eigenvalues can be analysed. Figure 12 shows the decay of the unresolved fluctuations as function of the number of modes utilised in the flow field reconstruction. It can be observed that a substantial number of proper orthogonal modes is required to represent sufficiently the fluctuation energy content. Further, the decay rate is lower for the entropy fluctuation representation at the high-pressure condition, which can be related to the smaller spatial size of the energetic perturbations.

Reconstructing the flow field by a truncated series of proper orthogonal modes filters low energetic fluctuations and therefore, not all details of the fluctuations are recovered. Comparing the root-mean-square variations contained in a reconstructed time series to the ones resolved in the large eddy simulation reveals the locations where disturbances are not well represented. The leading twenty vortical proper orthogonal modes represent the most characteristic large-scale vortical perturbations of the flow field (as proven in Fig. 13). Only the small-scale fluctuations in the shear layer between the exhaust gases and the cooling flow close to the outer circumference are less well captured by the modal reconstruction. This can be attributed to the high-frequency nature of these fluctuations, which the leading modes of the proper orthogonal decomposition do not contain (as shown in Fig. 14). For both operating conditions, the recovered vorticity spectrum with a 


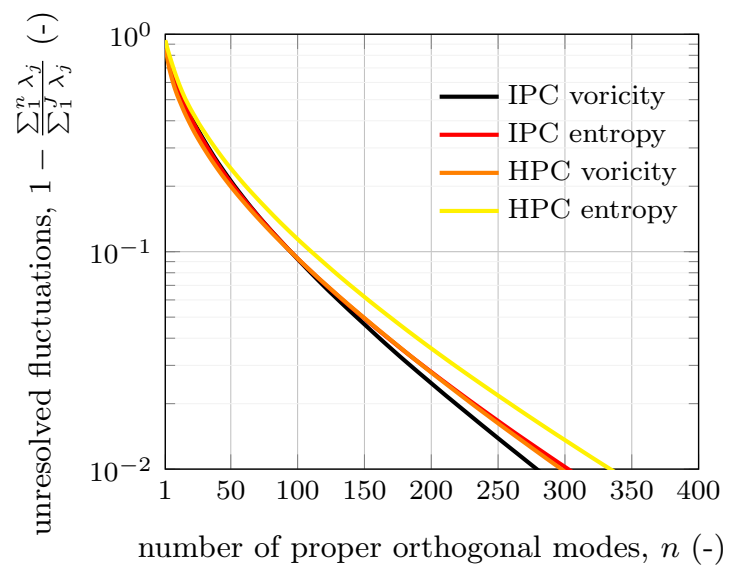

Fig. 12 Unresolved fluctuations as function of proper orthogonal modes considered.

few vortical modes matches the low-frequency range (below $1 \mathrm{kHz}$ ) contained in the full simulations.

Using twenty proper orthogonal modes to describe the entropy perturbations at the combustor exit leads to errors below $20 \%$ in the bulk flow for the intermediate pressure condition. Figure 13 illustrates that highest errors are committed in the outer boundary layer, where the perturbation amplitudes are small. Further, the low-frequent perturbation amplitudes are captured, as shown in Fig. 14, even with a few proper orthogonal modes. For the high-pressure condition, the errors to represent root-mean-square statistics of the perturbations with twenty proper orthogonal modes are high $(>20 \%)$, where the perturbation amplitudes are low. Figure 4 showed that this occurs at mid-passage in the combustor exit plane for this operating condition. Therefore, the spectral amplitudes of the low-frequent perturbations are lower as compared with the intermediate pressure condition and Fig. 14 shows that these are under-resolved when using only a few proper orthogonal modes.

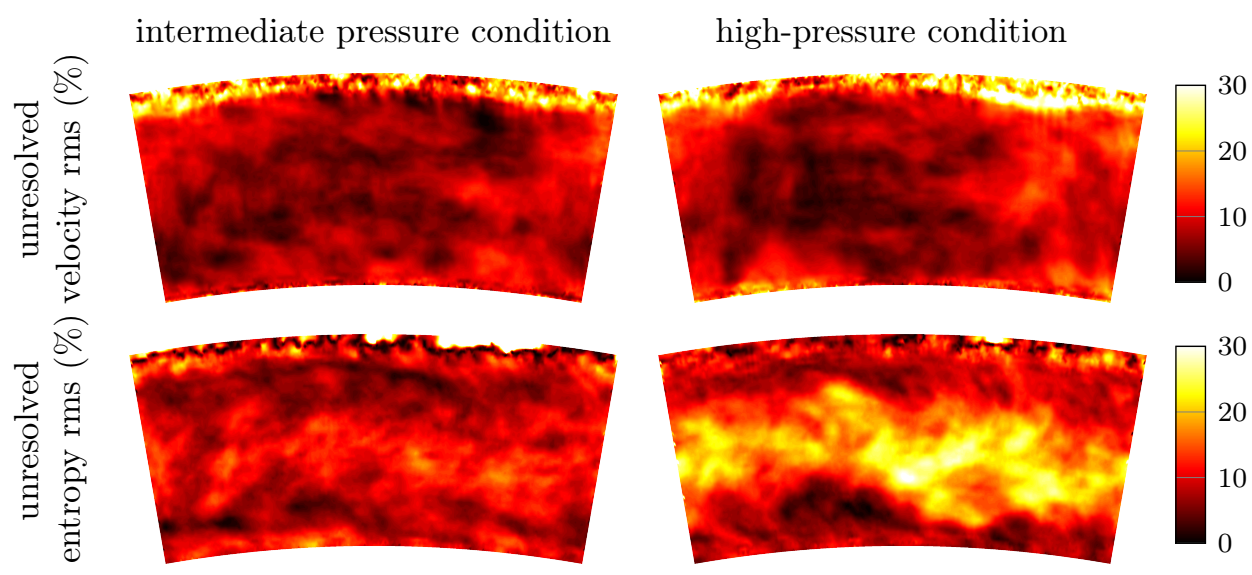

Fig. 13 The relative error between the root-mean-square fluctuations recovered (using twenty POD modes) and the resolved fluctuations with the LES is shown. 
intermediate pressure condition
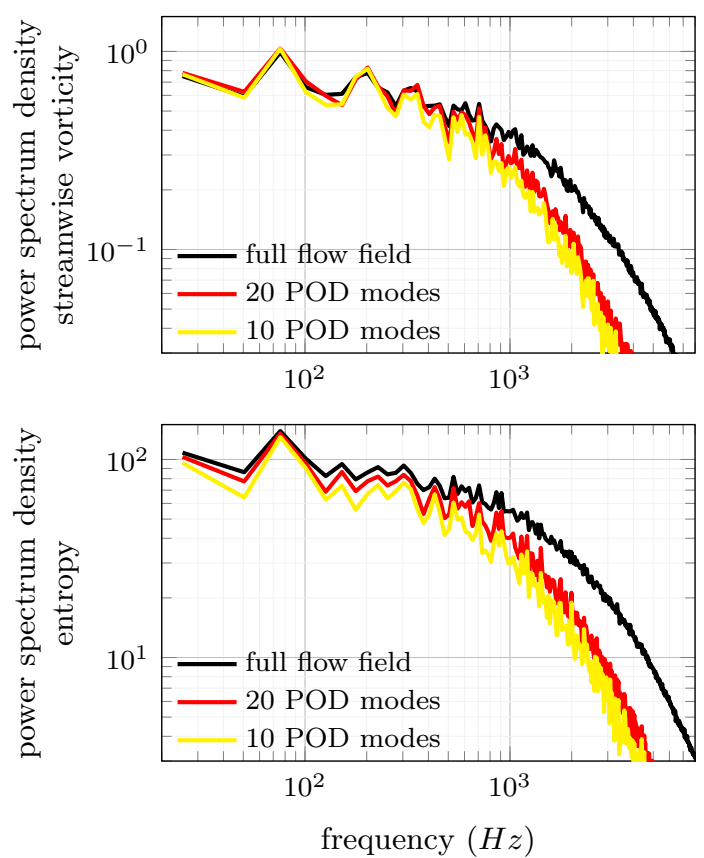

high-pressure condition
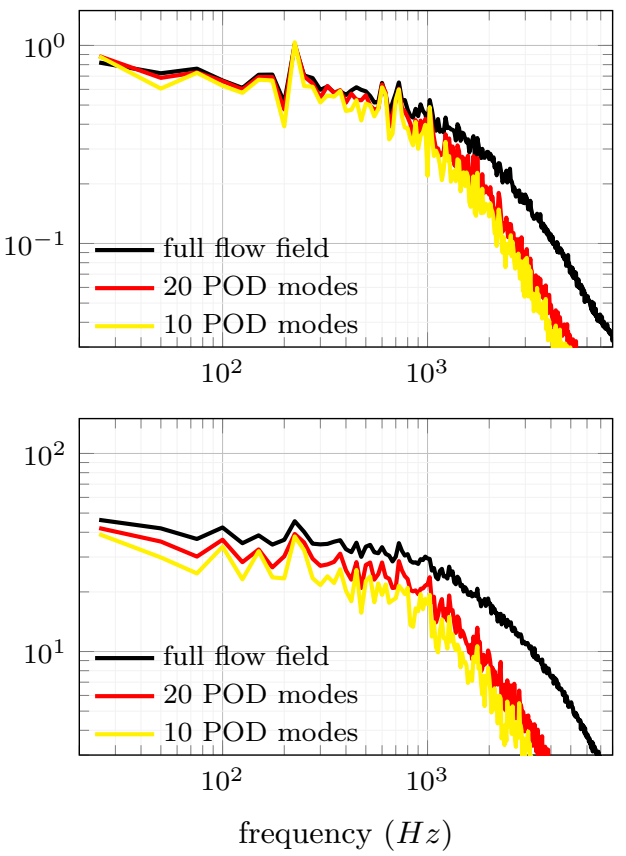

Fig. 14 The perturbation energy spectrum of the streamwise vorticity and entropy waves are shown when reconstructed with proper orthogonal modes.

\section{Conclusions}

We simulated the combustion process of a realistic, annular combustor in a single and double sector and analysed the coherent flow properties at its exit for near-ground operating conditions. Swirl, centred on the injectors, persists from the combustor inlets to the exit and dominates the outflow character independently of the operating condition. The air/fuel ratio determines the flame length and hence, the location of high heat release fluctuations. For a long flame, the entropy waves arise in the proximity of walls due to the mixing process of hot combustion gasses and cooling flow and convect along the walls towards the turbine. Hence, the cooling flow was found to be an essential factor governing the entropy wave amplitudes at the combustor exit at the high-pressure operating condition. At the intermediate pressure condition, entropy perturbations are generated primarily at the lean, main flame and its interaction with the pilot. A shorter main flame induces coherent entropy perturbations foremost at the inner shear layer separating the main from the pilot flame. These entropy waves propagate with the bulk flow in the annular channel.

The simulation of the double sector revealed a dominant out-of-phase oscillation of the axial vorticity between the sectors at combustor exit. Therefore, the simulation of multiple sectors is essential to capture the vorticity fluctuation statistics at the combustor exit.

At the combustor exit, the entropy and vorticity waves exhibit broadband and non-linear character. Thus, 
this analysis of the turbine inlet conditions suggests that entropy waves cannot be realistically modelled as planar waves or pulsation of the time-averaged temperature field. Rather, their size, location, and temporal evolution need to be considered. A significant number of proper orthogonal modes needs to be considered to capture over $90 \%$ of the velocity fluctuations. However, fewer (10 to 20$)$ proper orthogonal modes are required to represent the low-frequency content satisfactory, while high-frequency fluctuations near the walls remain under-resolved.

\section{Acknowledgments}

The authors wish to express their sincere gratitude to Rolls-Royce plc for permission to publish this paper. This work was conducted within the EU Horizon 2020 Framework Research Programme - Clean Sky (CORNET-CORE Noise Technologies), project number: H2020-CS2-CFP01-2014-01/ 686332.

\section{References}

[1] Morgans, A. S., and Duran, I., "Entropy noise: A review of theory, progress and challenges," International Journal of Spray and Combustion Dynamics, Vol. 8, No. 4, 2016, pp. 285-298. doi:10.1177/1756827716651791.

[2] Funke, S., Siller, H. A., Hage, W., and Lemke, O., "Microphone-Array Measurements of a Rolls-Royce BR700 Series Aeroengine in an Indoor Test-Bed and Comparison with Free-Field Data," 20th AIAA/CEAS Aeroacoustics Conference, 2014, p. 3070. doi:10.2514/6.2014-3070.

[3] Tester, B. J., "Engine Noise Source Breakdowns from an Improved Inverse Method (AFINDS) of processing Phased Array Measurements," 20th AIAA/CEAS Aeroacoustics Conference, 2014, p. 3067. doi:10.2514/6.2014-3067.

[4] Ekoule, C. M., Tester, B. J., Funke, S., and Richter, C., "Development of an Improved Core Noise Prediction Method for Long-cowl Engines," 2018 AIAA/CEAS Aeroacoustics Conference, 2018, p. 4090. doi:10.2514/6.2018-4090.

[5] Marble, F., and Candel, S., "Acoustic Disturbance from Gas Non-Uniformities convected through a Nozzle," Journal of Sound and Vibration, Vol. 55, No. 2, 1977, pp. 225-243. doi:10.1016/0022-460X(77)90596-X.

[6] Jacobi, S., Mazzoni, C., Rosic, B., and Chana, K., "Investigation of Unsteady Flow Phenomena in First Vane Caused by Combustor Flow With Swirl," Journal of Turbomachinery, Vol. 139, No. 4, 2017, p. 041006. doi:10.1115/1.4035073.

[7] De Domenico, F., Rolland, E. O., and Hochgreb, S., "Detection of Direct and Indirect Noise Generated by Synthetic Hot Spots in a Duct," Journal of Sound and Vibration, Vol. 394, 2017, pp. 220-236. doi:10.1016/j.jsv.2017.01.004.

[8] Poinsot, T., "Prediction and Control of Combustion Instabilities in Real Engines," Proceedings of the Combustion Institute, Vol. 36, No. 1, 2017, pp. 1-28. doi:10.1016/j.proci.2016.05.007. 
[9] Mu, Z., Michel, U., Steger, M., Ashcroft, G., Kennepohl, F., and Thiele, F., "Sound Emission of a Turbine Stage due to an Azimuthally Periodic Mean Temperature," International Journal of Aeroacoustics, Vol. 15, No. 1-2, 2016, pp. 162-179. doi:10.1177/1475472X15627408.

[10] Leyko, M., Nicoud, F., and Poinsot, T., "Comparison of Direct and Indirect Combustion Noise Mechanisms in a Model Combustor," AIAA Journal, Vol. 47, No. 11, 2009, p. 2709. doi:10.2514/1.43729.

[11] Duran, I., and Moreau, S., "Solution of the Quasi-One-Dimensional Linearized Euler Equations using Flow Invariants and the Magnus Expansion," Journal of Fluid Mechanics, Vol. 723, 2013, pp. 190-231. doi:10.1017/ jfm.2013.118.

[12] Cumpsty, N., and Marble, F., "The Interaction of Entropy Fluctuations with Turbine Blade Rows; a Mechanism of Turbojet Engine Noise," Proceedings of the Royal Society of London A: Mathematical, Physical and Engineering Sciences, Vol. 357, The Royal Society, 1977, pp. 323-344. doi:10.1098/rspa.1977.0171.

[13] Knobloch, K., Guerin, S., Holewa, A., Mahmoudi-Larimi, Y., Hynes, T., and Bake, F., "Noise Transmission Characteristics of a High Pressure Turbine Stage," 22nd AIAA/CEAS Aeroacoustics Conference, 2016, p. 3001. doi:10.2514/6.2016-3001.

[14] Livebardon, T., Moreau, S., Gicquel, L., Poinsot, T., and Bouty, E., "Combining LES of Combustion Chamber and an Actuator Disk Theory to Predict Combustion Noise in a Helicopter Engine," Combustion and Flame, Vol. 165, 2016, pp. 272-287. doi:10.1016/j.combustflame.2015.12.012.

[15] Mishra, A., and Bodony, D. J., "Evaluation of Actuator Disk Theory for Predicting Indirect Combustion Noise," Journal of Sound and Vibration, Vol. 332, No. 4, 2013, pp. 821-838. doi:10.1016/j.jsv.2012.09.025.

[16] Holewa, A., Lesnik, S., Ashcroft, G., and Guérin, S., "CFD-Based Investigation of Turbine Tonal Noise Induced by Steady Hot Streaks," International Journal of Turbomachinery, Propulsion and Power, Vol. 2, No. 1, 2017, p. 3. doi:10.3390/ijtpp2010003.

[17] Gaetani, P., and Persico, G., "Hot Streak Evolution in an Axial HP Turbine Stage," International Journal of Turbomachinery, Propulsion and Power, Vol. 2, No. 2, 2017, p. 6. doi:10.3390/ijtpp2020006.

[18] Ceci, A., Gojon, R., and Mihaescu, M., "Large Eddy Simulations for Indirect Combustion Noise Assessment in a Nozzle Guide Vane Passage," Flow, Turbulence and Combustion, 2018, pp. 1-13. doi:10.1007/s10494-018-9964-9.

[19] Ihme, M., "Combustion and Engine-Core Noise," Annual Review of Fluid Mechanics, Vol. 49, 2017, pp. $277-310$. doi:10.1146/annurev-fluid-122414-034542.

[20] Langella, I., Chen, Z., Swaminathan, N., and SK, S., "Large-Eddy Simulation of Reacting Flows in Industrial Gas Turbine Combustor," Journal of Propulsion and Power, Vol. 34, No. 5, 2018, pp. 1269-1284. doi:10.2514/1.B36842. 
[21] Ruan, S., Swaminathan, N., and Darbyshire, O., "Modelling of Turbulent lifted Jet Flames using Flamelets: a priori Assessment and a posteriori Validation," Combustion Theory and Modelling, Vol. 18, No. 2, 2014, pp. 295-329. doi:10.1080/13647830.2014.898409.

[22] Chen, Z., Ruan, S., and Swaminathan, N., "Large Eddy Simulation of Flame Edge Evolution in a Spark-Ignited Methane-Air Jet," Proceedings of the Combustion Institute, Vol. 36, 2017, pp. 1645-1652. doi:10.1016/j.proci. 2016.06.023.

[23] Pierce, C. D., and Moin, P., "A Dynamic Model for Subgrid-Scale Variance and Dissipation Rate of a Conserved Scalar," Physics of Fluids, Vol. 10, No. 12, 1998, pp. 3041-3044. doi:10.2514/6.2014-3067.

[24] Langella, I., and Swaminathan, N., "Unstrained and Strained Flamelets for LES of Premixed Combustion," Combustion Theory and Modelling, Vol. 20, No. 3, 2016, pp. 410-440. doi:10.1080/13647830.2016.1140230.

[25] Dunstan, T. D., Minamoto, Y., Chakraborty, N., and Swaminathan, N., "Scalar Dissipation Rate Modelling for Large Eddy Simulation of Turbulent Premixed Flames," Proceedings of the Conbustion Institute, Vol. 34, 2013, pp. 1193-1201. doi:10.1016/j.proci.2012.06.143.

[26] Langella, I., Swaminathan, N., Gao, Y., and Chakraborty, N., "Assessment of Dynamic Closure for Premixed Combustion Large Eddy Simulation," Combustion Theory and Modelling, Vol. 19, 2015, pp. 628-656. doi: $10.1080 / 13647830.2015 .1080387$.

[27] Schmehl, R., Maier, G., and Wittig, S., "CFD Analysis of Fuel Atomization, Secondary Droplet Breakup and Spray Dispersion in the Premix Duct of a LPP Combustor," 8th International Conference on Liquid Atomization ES Spray Systems, ICLASS, 2000.

[28] Anand, M., Eggels, R., Staufer, M., Zedda, M., and Zhu, J., "An Advanced Unstructured-Grid Finite-Volume Design System for Gas Turbine Combustion Analysis," ASME 2013 Gas Turbine India Conference, American Society of Mechanical Engineers, 2013, pp. V001T03A003-V001T03A003. doi:10.1115/GTINDIA2013-3537.

[29] Pope, S. B., "Large-Eddy Simulation," Turbulent Flows, Cambridge University Press, Cambridge, 2000, Chap. 13.

[30] Doormaal, P., and Raithby, G. D., "Enhancements of the SIMPLE Method for Predicting Incompressible Fluid Flows," Numerical Heat Transfer, Vol. 7, No. 2, 1984, pp. 147-163. doi:10.1080/01495728408961817.

[31] Dagaut, P., and Cathonnet, M., "The Ignition, Oxidation, and Combustion of Kerosene: A Review of Experimental and Kinetic Modelling," Progress in Energy and Combustion Science, Vol. 32, 2006, pp. 48-92. doi:10.1016/j.pecs. 2005.10.003.

[32] Lilly, D., "The Representation of Small-Scale Turbulence in Numerical Simulation Experiments," IBM Scientific Computing Symposium on Environmental Sciences, edited by H. Goldstine, IBM, 1967, pp. V001T03A003V001T03A003. doi:10.5065/D62R3PMM. 
[33] Chatterjee, A., "An introduction to the proper orthogonal decomposition," Current science, 2000, pp. 808-817.

[34] Jolliffe, I., Principal component analysis, $2^{\text {nd }}$ ed., Springer Series in Statistics, Springer, 2011. 